\title{
Una propuesta didáctica para introducir la educación para la ciudadanía global en la enseñanza universitaria
}

\author{
A didactic proposal to introduce education for global citizenship in higher education
}

\section{A proposta didatico para introduzir a educação para a cidadania global no ensino superior}

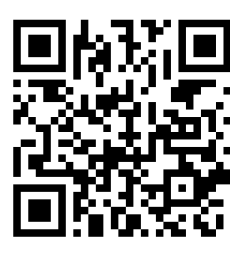

\section{María Martínez-Lirola?}

Universidad de Alicante

Alicante, España

maria.lirola@ua.es

http://orcid.org/0000-0002-6427-425X

Recibido • Received • Recebido: 07 / 07 / 2017
Corregido • Revised • Revisado: 08 / 11 / 2018
Aceptado • Accepted • Aprovado: 15 / 12 / 2018

Resumen: Una de las misiones de la Universidad del siglo XXI es formar profesionales que sepan dar respuestas a las demandas sociales. En este sentido, la educación para la ciudadanía global contribuye a producir transformaciones sociales, a la vez que potencia el aprendizaje integral del estudiantado al establecer relaciones entre lo que se enseña en las aulas y la realidad social. En este artículo se presentan una serie de actividades de temática social enmarcadas en el aprendizaje cooperativo en una asignatura de lengua inglesa. Se describirán dichas actividades, las principales competencias sociales que con ellas adquiere el estudiantado entre las que destacan la comunicación, la cooperación y la empatía. La propuesta didáctica que se presenta pretende que el estudiantado no adquiera solo conocimientos, sino que también adquiera competencias de modo que su formación sea integral. La metodología ha seguido un modelo cualitativo-descriptivo. Además, se preparó una encuesta con el fin de conocer la opinión del estudiantado sobre algunos aspectos del proceso de enseñanza-aprendizaje y saber si hay contribución en la formación de la ciudadanía global. Dicha encuesta se diseñó teniendo en cuenta las notas que la profesora había tomado en clase, al observar si el alumnado relacionaba las actividades sociales propuestas con la adquisición de competencias sociales. Los datos se recopilaron al final del cuatrimestre de manera anónima y se analizaron manualmente. Los resultados muestran que la mayoría del alumnado considera que se ha formado en ciudadanía global al tomar conciencia de realidades sociales distintas de la propia y empatizar con situaciones injustas. Este estudio revela que basar la asignatura en contenidos sociales no solo contribuye a la adquisición de competencias sino también a aumentar la formación social y global del alumnado.

Palabras claves: Educación para la ciudadanía global; competencias sociales; aprendizaje cooperativo; proceso de enseñanza-aprendizaje.

\footnotetext{
${ }^{1}$ University of South Africa.
} 
doi: http://dx.doi.org/10.15359/ree.23-2.15

URL: http://www.una.ac.cr/educare

CORREO: educare@una.cr

\begin{abstract}
One of the missions of the XXI century university is to train professionals to know how to respond to social demands. In this sense, education for global citizenship contributes to social transformations while enhancing the integral learning of students by establishing relationships between what is taught in classrooms and social reality. This article presents a series of social thematic activities framed in cooperative learning in an English language subject. These activities will be described, as well as the main social skills that students acquire with them, such as communication, cooperation, and empathy. The didactic proposal presented is intended for the students not only to acquire content, but also to acquire competences so that their formation is integral. The methodology used has followed a qualitative-descriptive model. Moreover, a survey was prepared in order to know students' opinion on some aspects of the teaching-learning process, and to find out if there was any contribution to the formation of global citizenship. This survey was designed taking into account the notes that the teacher had taken in class by observing if the students carried out a relationship between the proposed social activities and the acquisition of social competencies. The data were collected at the end of the semester anonymously, and analysed in a manual manner. The results show that the majority of the students believe that they have grown as global citizens by being aware of social realities distinct from their own, and empathize with unfair situations. This study reveals that basing the subject on social content not only contributes to the acquisition of competencies but also to increase students' social and global education.
\end{abstract}

Keywords: Education for global citizenship; social competences; cooperative learning; teachinglearning process.

Resumo: Uma das missões da Universidade do século XXI é formar profissionais que sabem como responder às demandas sociais. Neste sentido, a educação para a cidadania global ajuda a produzir a mudança social, reforçando simultaneamente o estudante geral aprender a estabelecer relações entre o que é ensinado nas salas de aula ea realidade social. Este artigo apresenta uma série de atividades sobre questões sociais enquadradas em uma metodologia cooperativa em um assunto do idioma Inglês são apresentados. essas atividades, as principais habilidades sociais que os estudantes adquiri-los entre os quais a comunicação, cooperação e empatia vai ser descrito. A proposta didática apresentada pretende-se que os alunos adquiram não apenas conhecimento mas também adquirir habilidades de modo que sua formação é completa. A metodologia utilizada seguiu um modelo qualitativo-descritivo. Além disso, uma pesquisa para saber a opinião dos estudantes sobre a metodologia utilizada foi preparada e saber se há alguma contribuição para a formação da cidadania global. Esta pesquisa foi concebida tendo em conta as notas que o professor tinha tomado em sala de aula, observando se os alunos realizaram uma relação entre as atividades sociais propostas e a aquisição de competências sociais. Os dados foram coletados no final do semestre anonimamente e analisados de forma manual. Os resultados mostram que a maioria dos alunos acredita que se formou como cidadania global por estar consciente das realidades sociais distintas das suas próprias e simpatizar com situações injustas. Este estudo revela que basear o sujeito em conteúdo social não só contribui para a aquisição de competências, mas também para aumentar a educação social e global dos alunos.

Palavras-chave: Educação para a cidadania global (ECG), habilidades sociais, a aprendizagem cooperativa, o processo de ensino-aprendizagem. 


\section{Introducción}

El carácter global de las sociedades actuales permite conocer realidades sociales y culturales distintas a la propia. La educación superior ha de contribuir a que el estudiantado pueda profundizar en manifestaciones culturales y en realidades que nada tienen que ver con lo que vive cada día (Francisco, 2010), introduciendo el componente intercultural en las aulas (Priegue y Leiva, 2012). Salir de la realidad local para adentrarse en la realidad global lleva consigo que el estudiantado crece como ciudadanía global al interesarse por conocer y por formarse en lo que ocurre en otros lugares del mundo.

Profundizar en diferentes realidades, incluyendo aquellas en las se observan marcadas injusticias o desigualdades, contribuirá a que el estudiantado amplíe su mirada y pueda desarrollar actitudes de empatía y solidaridad, a la vez que se adquieren competencias interculturales que facilitan el respeto a la diversidad y la apertura para entender otras formas de hacer (Baesler y Lauricella, 2014; Kruger, 2012).

Optamos, por tanto, por un modelo educativo que contribuya a la formación integral del estudiantado. En consecuencia, proponemos una educación que vaya más allá de la mera adquisición de conocimientos. Con el fin de conocer distintas realidades sociales, es necesario basar la enseñanza en competencias sociales. Siguiendo a Bisquerra y Pérez (2007, p. 72), entendemos la competencia social como"... la capacidad para mantener buenas relaciones con otras personas. Esto implica dominar las habilidades sociales, capacidad para la comunicación efectiva, respeto, actitudes pro-sociales, asertividad, etc.".

Tallón y Sikora (2011) dividen las competencias sociales en dos tipos: a) las que tienen que ver con la sintonía con los demás (incluye empatía, conciencia política, comunicación) y b) las que tienen que ver con la construcción de relaciones (incluye cooperación, liderazgo, influencia y gestión de conflictos). Estas competencias son importantes tanto para las relaciones que se establecen dentro del aula como para las relaciones que se establecen en distintos contextos sociales.

Con el fin de que el estudiantado pueda adquirir las competencias sociales señaladas, se diseñarán diferentes actividades gracias a las que se pueda profundizar en distintas realidades sociales/culturales a la vez que se trabaja por competencias. Nos parece fundamental promover metodologías activas que permitan al estudiantado dar respuesta a las demandas del mercado laboral, entre las que destaca poder trabajar con otras personas de manera cooperativa. Por tanto, la metodología que se empleará en el aula estará enmarcada por los principios del aprendizaje cooperativo $(A C)$, con el fin de que el estudiantado trabaje en colaboración con el fin de lograr un objetivo común (D.W. Johnson y F. P. Johnson, 2009; D. W. Johnson y R. T. Johnson, 2009; Prieto, 2007). 
doi: http://dx.doi.org/10.15359/ree.23-2.15

URL: http://www.una.ac.cr/educare

CORREO: educare@una.cr

En el aprendizaje cooperativo distintas personas trabajan en colaboración con el fin de lograr un objetivo común de modo que comparten la responsabilidad del resultado final, además de aprender haciendo durante todo el proceso de enseñanza-aprendizaje (Prieto, 2007). Es decir, el estudiantado aprende de manera conjunta con el fin de aprender y de que el resto de las personas que integran el grupo también aprendan.

Siguiendo a D. W. Johnson, R. T. Johnson y Holubec (1998), para que el aprendizaje sea cooperativo, se deben generar las siguientes condiciones: interdependencia positiva (el estudiantado comparte tanto los recursos para desarrollar las actividades como las metas); responsabilidad individual (es fundamental que cada persona que integra el grupo se responsabilice de la tarea y de su resultado final); comunicación cara a cara (en los grupos se potencia el apoyo entre los distintos miembros, así como el intercambio de materiales e información); habilidades interpersonales (se potencia el desarrollo de competencias sociales como la comunicación, la resolución de conflictos o la toma de decisiones) y reflexión sobre el trabajo (el estudiantado ha de evaluar el trabajo realizado y el logro de objetivos).

Seleccionar el aprendizaje cooperativo en el proceso educativo facilita la adquisición de competencias, debido a que el estudiantado comparte con sus iguales mientras aprende, de modo que se favorece la interacción, la toma de decisiones consensuadas, la escucha activa o el respeto a la diversidad (Martínez-Lirola, 2013; Saura y Del Valle Antolín, 2012). De esta forma, el resultado final depende del trabajo realizado y de las decisiones tomadas durante el proceso de enseñanza-aprendizaje, donde cada persona tiene una responsabilidad que repercute en la calidad del resultado final.

El AC es muy apropiado para potenciar los principios de la educación para la ciudadanía global (ECG), debido a que se concede importancia a interactuar con otras personas con el fin de profundizar en temas sociales como son la diversidad étnica y el género en el caso de este artículo. La pertinencia del AC para la adquisición de competencias sociales está también directamente relacionada con la ECG debido al papel central que se concede a que el estudiantado tome conciencia ciudadana de distintas realidades sociales que afectan a las personas en distintos lugares del mundo; se empatiza con realidades sociales difíciles y se potencia en todo momento la comunicación en los grupos cooperativos, con el fin de poder profundizar y comprender mejor las realidades objeto de análisis.

El objetivo principal de esta investigación es diseñar una serie de actividades cooperativas que permitan al estudiantado profundizar en distintas realidades sociales con el fin de crecer como ciudadanía global, a la vez que se adquieren distintas competencias sociales. De este modo se podrán superar modelos etnocéntricos que conceden más valor e importancia a la cultura propia que a las demás (Wang, Young, Wilhite y Marczyk, 2011). Otro objetivo es establecer puentes entre lo que se enseña en las aulas y lo que ocurre en la sociedad, al trabajar con textos reales de temática social. 
Pretendemos ofrecer una propuesta educativa que ayude al estudiantado a avanzar como ciudadanía global al tomar conciencia de las desigualdades sociales que existen en distintos lugares del mundo, al ver en las diferencias una oportunidad para enriquecerse y avanzar en el respeto a la diversidad y al comprometerse en la transformación tanto de la realidad local como la global. Por tanto, el estudiantado ha de asumir un papel activo en el proceso de enseñanzaaprendizaje y ha de tener un compromiso activo en la transformación de un mundo mejor.

La principal pregunta que nos planteamos en esta investigación esla siguiente: ¿Contribuyen la realización de actividades de temática social y el empleo de textos reales sobre cuestiones raciales y de género a la formación del alumnado universitario como ciudadanía global? Tras la realización de las actividades, se diseñó una encuesta con el fin de conocer la opinión del estudiantado sobre distintos aspectos del proceso de enseñanza-aprendizaje e identificar la contribución de la experiencia en la formación del alumnado como ciudadanía global.

\section{Estado de la cuestión}

Trabajar en el aula universitaria de forma que el estudiantado avance como ciudadanía global para que se respete la diversidad y los derechos humanos requiere de una propuesta pedagógica que enmarque el proceso de enseñanza aprendizaje. Hemos optado por la educación para la ciudadanía global (Boni y Calabuig, 2017; Boni, Lopez-Fogues y Walker, 2016) por la importancia que concede a la diversidad, la interculturalidad y a la justicia social en el proceso de enseñanza-aprendizaje. Se concede mucha importancia al acercamiento a otras culturas, al entendimiento de las diferencias culturales, a la interculturalidad, a los derechos humanos y a la paz (Cantón Mayo, Valle Flórez, Arias Gago, Baelo Álvarez y Cañón Rodríguez, 2010; Vinagre, 2014).

Así, los objetivos de este modelo educativo son: a) contribuir a que la educación pueda producir transformaciones sociales debido a que las personas asumen un compromiso activo en la transformación social que lleva consigo avanzar en la construcción de un mundo más justo y más humano; b) potenciar el aprendizaje global de modo que la educación vaya más allá de la mera adquisición de conocimientos sino que se potencia tomar conciencia de los cambios que es necesario llevar a cabo en la sociedad con el fin de se produzcan transformaciones. Por tanto, se pretende que el estudiantado avance en la perspectiva de la ciudadanía global, tal y como señalan Barahona, Gratacós y Quintana (2013, p. 12):

La Educación para una ciudadanía global (ECG) es el fruto de una larga tradición social y pedagógica que cree en el poder transformador de la educación, y en ese sentido no solamente plantea unos medios didácticos, sino, sobre todo, plantea unos objetivos políticos del proceso educativo-socializador al modelo de sociedad que queremos construir. 
doi: http://dx.doi.org/10.15359/ree.23-2.15

URL: http://www.una.ac.cr/educare

CORREO: educare@una.cr

La ECG debe ayudar al estudiantado a desarrollar una identidad global y un interés por la comunidad global, así como una conexión con personas de todo el mundo. De este modo, el estudiantado avanza como ciudadanía cosmopolita a la vez que mantiene una unión con su familia y su cultura (Moss, Barletta, Chamorro y Mizuno, 2015; Porto, 2015). Además, en este marco, se trabaja por la inclusión de todas las personas con independencia de su sexo, clase social, raza o condición sexual, entre otros. Así, la ECG trata de contribuir al empoderamiento de los grupos sociales más desfavorecidos, a la vez que avanza en la formación en valores como la paz, la solidaridad, la justicia o el respeto a la diversidad, entre otros.

Una idea fundamental para la ECG es trabajar con temas globales (global issues) con el fin de profundizar en los cambios sociales y en los retos que tiene la sociedad, como, por ejemplo, la violencia de género o el racismo. Se potencia, por tanto, reflexionar sobre las realidades de otras personas con el fin de ampliar la mirada y potenciar una enseñanza basada en valores y competencias sociales. El empleo de materiales con los que se potencien los principios de la ECG, así como de una metodología cooperativa, permite que el estudiantado de distintos grupos raciales y étnicos pueda desarrollar actitudes democráticas a la vez que promueve la interacción de igual a igual. En este sentido, el profesorado juega un papel fundamental pues ha de educar personas abiertas a otras culturas, capaces de respetar las diferencias y de comprometerse con la realidad social (Rosales, 2015).

Este enfoque educativo ofrece al estudiantado la oportunidad de adquirir conocimientos, actitudes y destrezas que le permitan desenvolverse tanto en su entorno cultural como en los de otras culturas. Así, el estudiantado es capaz de adaptarse a las necesidades de un mundo globalizado en el que se encuentran personas de distintas etnias, culturas, lenguas, religiones, etc. La ECG nos parece un enfoque adecuado en la clase de inglés como lengua extranjera, porque permite al estudiantado comprender cómo la vida de sus comunidades y países influye en otras naciones y como determinados hechos internacionales afectan su cotidianidad, es decir, se toma conciencia de que hay interdependencia entre naciones.

Hemos optado por la ECG porque, a pesar de todos los avances que se están llevando a cabo en el mundo gracias a la globalización, sigue habiendo seres humanos discriminados y maltratados. Por tanto, este enfoque apuesta por la ciudadanía global en la que ocupa un lugar fundamental el respeto a la diversidad, hecho que contribuye al fomento de la convivencia pacífica. Se adquiere una visión mundialista de la realidad al establecer relaciones entre lo local y lo global.

\section{Contexto, participantes y metodología}

Las actividades que se presentan en la sección siguiente se enmarcan en la asignatura obligatoria Lengua Inglesa V que se imparte en tercero del grado en Estudios Ingleses de la Universidad de Alicante. En esta asignatura el estudiantado ha de desarrollarse en las cinco 
destrezas (lectura, habla, escritura, escucha e interacción) con el fin de alcanzar un nivel C1 dentro del Marco Común Europeo de Referencia de las Lenguas, propuesto por el Consejo de Europa en el año 2001. La asignatura es de seis créditos, es decir, el estudiantado ha de asistir a 60 horas presenciales, además de trabajar de manera individual y grupal durante 90 horas fuera del aula. Las cuatro horas presenciales semanales se dedican a la enseñanza de la gramática, de la escritura académica y en las otras dos horas el estudiantado ha de preparar una presentación oral y un debate cooperativo con el fin de trabajar las destrezas orales.

Durante el curso académico 2016-2017, la asignatura contó con 102 personas matriculadas, de las cuales el $87 \%$ eran mujeres frente al $13 \%$ que eran hombres. El alumnado tenía entre 20 y 22 años. Todo el estudiantado ha estudiado inglés tanto en secundaria como en los dos cursos anteriores del grado, por lo que cuando cursan esta asignatura su nivel es B2 (Consejo de Europa, 2001). La mayor parte del estudiantado quiere dedicarse a la enseñanza del inglés en secundaria, pero también hay estudiantes con interés en el comercio exterior y en la enseñanza del español como lengua extranjera.

Se optó por emplear el aprendizaje cooperativo debido al alto número de personas matriculadas en la asignatura. Así, el estudiantado se organizó en pequeños grupos de cinco personas, con el fin de trabajar de manera cooperativa durante todo el cuatrimestre. Se diseñaron unas actividades de temática social enmarcadas en el aprendizaje cooperativo, con el fin de que el estudiantado se aproximara al racismo y la discriminación por sexo a través de los textos y, a la vez, para que adquiera distintas competencias que contribuyen a la formación global. La interacción se potencia en todo momento del proceso de enseñanza-aprendizaje. Debido a las limitaciones de espacio de este artículo, nos vamos a centrar en describir solo dos de las actividades llevadas a cabo (vid. propuesta de actividades) y en posteriores estudios presentaremos otras actividades.

La metodología y técnicas utilizadas han seguido un modelo cualitativo-descriptivo con carácter exploratorio, pues la profesora tomó notas basadas en la observación sobre cómo se desarrollaba el proceso de enseñanza-aprendizaje en cada clase. Todo el alumnado matriculado en la asignatura participó en las actividades propuestas. Al final del proceso de enseñanza-aprendizaje el estudiantado respondió a una encuesta con el fin de expresar su opinión de manera anónima sobre determinados aspectos relacionados con la propuesta didáctica planteada durante el cuatrimestre (ver apéndice A). La encuesta proporciona datos cuantitativos que ayudan a identificar la contribución de la experiencia didáctica en la formación del estudiantado como ciudadanía global.

Dicha encuesta se basó en las notas que la profesora tomó en cada clase, teniendo en cuenta que las distintas actividades se diseñaron para que el alumnado adquiriera competencias sociales y se formara de manera integral. Las preguntas se diseñaron para hacer reflexionar al 
doi: http://dx.doi.org/10.15359/ree.23-2.15

URL: http://www.una.ac.cr/educare

CORREO: educare@una.cr

alumnado sobre los principales aspectos que la profesora había tenido en cuenta tanto en la planificación de la metodología como en el diseño de las actividades. Los datos se recopilaron al final del cuatrimestre de manera anónima y se analizaron de manera manual.

\section{Propuesta de actividades}

El hecho de que la asignatura en la que se lleva a la práctica la propuesta educativa que se presenta en este artículo sea una lengua extranjera hace posible el uso de materiales y de temas que estén relacionados con lo que ocurre en la sociedad. Así, el estudiantado puede reflexionar sobre los temas seleccionados de modo que mejora su capacidad de análisis y el pensamiento crítico. Además, ha de tomar conciencia de que puede contribuir a la transformación social si es consciente de su papel activo en las aulas y en la sociedad.

El empleo de textos con contenido social en una asignatura de lengua extranjera puede contribuir a trabajar distintos tipos de competencias, incluidas las sociales. Estos textos son adecuados para trabajar de manera cooperativa y motivar a los grupos de estudiantes a interaccionar en el aula, cuestión esencial en una pedagogía activa. La profesora decidió seleccionar dos temas globales importantes como son el racismo y cuestiones de desigualdad de género, incluida la violencia de género (vid. las secciones que siguen dedicadas a las actividades concretas). En la siguiente tabla, se presentan las dos temáticas seleccionadas y las distintas actividades que se llevaron a cabo para alcanzar el propósito relacionado con la ciudadanía global que se perseguía con ellas. La profesora basó sus observaciones en el modo en que el alumnado llevaba a cabo las actividades que se indican en la Tabla 1.

Tabla 1: Temáticas y actividades seleccionadas para fomentar la ciudadanía global

\begin{tabular}{|c|c|c|}
\hline Temática & Actividades & Relaciones con el desarrollo de la ciudadanía global \\
\hline $\begin{array}{l}\text { Temas raciales } \\
\text { en el aula }\end{array}$ & $\begin{array}{l}\text { - Selección de noticias sobre inmigración. } \\
\text { - Análisis multimodal de textos. } \\
\text { - Escritura de un ensayo académico. } \\
\text {-Participación en debate cooperativo. }\end{array}$ & $\begin{array}{l}\text { - Reflexionar sobre realidades sociales distintas de } \\
\text { la propia: la de las personas inmigrantes. } \\
\text { - Deconstruir los mensajes y la ideología que la } \\
\text { prensa transmite sobre la inmigración. } \\
\text { - Reconocer prejuicios y estereotipos. }\end{array}$ \\
\hline $\begin{array}{l}\text { Temas de } \\
\text { género en el } \\
\text { aula }\end{array}$ & $\begin{array}{l}\text { - Preparación de glosario de términos } \\
\text { sobre género y violencia de género. } \\
\text { - Reflexión basada en un video sobre género. } \\
\text { - Búsqueda de textos, análisis multimodal y } \\
\text { resumen de los resultados. } \\
\text { - Participación en debate. } \\
\text { - Escritura de carta formal. }\end{array}$ & $\begin{array}{l}\text { - Reflexionar sobre la situación de las mujeres en } \\
\text { distintos lugares del planeta. } \\
\text { - Deconstruir el discurso de la publicidad sobre las } \\
\text { mujeres. } \\
\text { - Debatir sobre la violencia de género y la } \\
\text { cosificación de las mujeres en la publicidad } \\
\text { como fenómenos globales. }\end{array}$ \\
\hline
\end{tabular}

Nota: Elaboración propia. 
En las secciones que siguen, se explica el modo en que se trabajó en el aula con cada una de las actividades seleccionadas. Todas ellas contribuyen a generar las distintas condiciones que hacen que el aprendizaje sea cooperativo (presentadas en la introducción de este artículo), debido a que para que el resultado final de cada tarea sea satisfactorio es necesario que cada estudiante se implique en el proceso. Además, tal y como se puede observar en las secciones siguientes, en todas las actividades se potencia la comunicación escrita u oral y la cooperación entre el alumnado. Las tareas propuestas también contribuyen al desarrollo de las habilidades interpersonales por tratarse de cuestiones fundamentales para el desarrollo de distintas destrezas en el aprendizaje de una lengua extranjera.

\section{Propuesta para trabajar temas raciales en el aula}

El primer tema relacionado con la ECG que la profesora decidió trabajar en el aula se relaciona con la inmigración y el racismo. De esta forma, se establece una relación entre lo que se enseña en las aulas y la realidad social (Martínez-Lirola, 2014). Trabajar con temas globales permite profundizar en distintas realidades sociales, a la vez que se aprende vocabulario relacionado. De hecho, nuestra propuesta educativa se basa en textos con el fin de obtener información sobre el contexto presente en el texto y sobre el contexto social en que los textos se producen y se leen (De Sylva Joyce y Feez, 2012; Martin y Rose, 2012).

La profesora pidió al estudiantado que buscara en distintos periódicos de habla inglesa noticias relacionadas con la inmigración, con el fin de observar la temática de dichas noticias y el modo en que los grupos inmigrantes eran representados comparados con el grupo mayoritario. Una vez que el estudiantado había recopilado las noticias, al menos diez de cada grupo tenía que hacer un esquema con las principales características observadas. Se nombró un sujeto portavoz en cada grupo, con el fin de compartir con toda la clase el esquema preparado. Una vez que todos los grupos habían participado, se pidió al estudiantado que preparara un listado con vocabulario y algunos campos semánticos relacionados con la inmigración. Se emplearon diccionarios electrónicos para buscar las palabras que no conocían.

La mayoría de los textos que el estudiantado había recopilado eran multimodales, es decir, presentaban más de un modo de comunicación y la imagen ocupaba un papel importante en la transmisión del significado. Con el fin de que el estudiantado pudiera profundizar en las noticias recopiladas, la profesora explicó el modelo de gramática visual propuesto por Kress y van Leeuwen (2006), presentado especial atención en los principios de composición: el valor de la información (dónde aparece la información conocida y la información nueva en la imagen); cuál es el elemento más sobresaliente y finalmente los márgenes que sirven para conectar elementos que van unidos. Profundizar en este modelo permitía al estudiantado leer no solo el texto escrito sino también la imagen y entender, de esta forma, los distintos elementos que componen el texto multimodal como un todo, en el que los distintos elementos que lo componen no son elegidos al azar sino con una función comunicativa determinada. 
doi: http://dx.doi.org/10.15359/ree.23-2.15

URL: http://www.una.ac.cr/educare

CORREO: educare@una.cr

Una vez explicada la gramática visual, el estudiantado tenía que analizar los textos recopilados teniendo en cuenta sus principios. Este análisis permitía al estudiantado sistematizar el tipo de imágenes que el periodismo selecciona para transmitir la realidad migratoria y poder sacar algunas conclusiones sobre la ideología que hay detrás de cada una de las elecciones lingüísticas y visuales que aparecen en los textos analizados. Cuando el análisis estuvo terminado, la profesora pidió al estudiantado que organizara sus ideas para de presentarlas en un texto escrito.

A continuación, la profesora explicó la estructura del ensayo académico de modo que el estudiantado pudiera presentar sus ideas basadas en el análisis de un modo organizado, en un ensayo argumentativo con una clara introducción, unos párrafos donde exponer sus ideas principales y unas conclusiones. Mejorar la escritura académica es sin duda uno de los objetivos de la asignatura. Por tanto, la profesora sistematizó los principales recursos cohesivos en inglés (referencia, sustitución, elipsis, conjunción y coherencia léxica) y los ilustró con ejemplos de textos reales de modo que el estudiantado pudiera observar cómo estos recursos contribuyen a que el texto sea cohesivo y a que las ideas se expresen de un modo claro y ordenado, evitando repeticiones y redundancias.

El estudiantado tenía que preparar un ensayo cooperativo basado en el análisis de los textos multimodales analizados sobre inmigración. El hecho de optar por la escritura cooperativa implica que todo el estudiantado es responsable del ensayo final. Además, de esta forma, el estudiantado con más nivel en la destreza escrita contribuye a que la mejore el estudiantado que tiene más dificultades para la escritura académica.

Seguidamente, se estableció un debate para profundizar en el modo en que la prensa transmite la realidad del fenómeno migratorio. Cada miembro del grupo tenía que participar al menos una vez compartiendo su opinión sobre la realidad migratoria basada en el análisis de textos llevado a cabo y en las ideas que cada grupo había trabajado para preparar el ensayo. Una vez que cada grupo había compartido sus ideas, la profesora formuló algunas preguntas relacionadas con el tema con el fin de que el estudiantado profundizara en los principios de la ECG: ¿qué entiendes por cultura?, ¿cuántas diferencias culturales conoces?, enumera algunos prejuicios racionales que observas en la sociedad y en los textos de prensa analizados, ¿qué derechos y deberes lleva consigo pertenecer a la ciudadanía de un país?, ¿aparecen los grupos inmigrantes representados en los textos que has analizado como ciudadanía?

Al acabar el debate, la profesora precisó la importancia de ampliar la mirada en cuestiones relacionadas con personas de otras culturas para comprender las razones por las que las personas dejan sus países de origen. Se señaló la necesidad de avanzar como ciudadanía global y comprometida, por lo que se han de rechazar los prejuicios y estereotipos que muchas veces nos transmiten los medios de comunicación en general y la prensa en particular. Finalmente, la 
profesora precisó que los textos son producto de la sociedad en la que se enmarcan, de modo que las noticias de prensa analizadas son el resultado del contexto social en que se enmarcan por lo que para que la realidad migratoria de transmitiera de otra manera sería necesario realizar cambios político-sociales relacionados con la inmigración.

\section{Propuesta para trabajar temas de género en el aula}

El segundo tema global seleccionado tiene que ver con la situación de las mujeres en distintos lugares del planeta y con la imagen que la publicidad transmite sobre ellas. Esto implica cuestionarse determinados estereotipos sexistas que están presentes en todas las sociedades, pero sin duda, el primer paso para superarlos es tomar conciencia de ellos.

En primer lugar, se pidió al estudiantado que preparara por grupos un glosario de términos relacionados con el género en general y la violencia de género en particular. A continuación, tenían que discutir en sus grupos las principales diferencias que observan entre mujeres y hombres tanto en su realidad como en otras realidades sociales. De esta forma, se invitaba al estudiantado a reflexionar sobre las principales formas de discriminación que afectan a la mujer, de ahí la importancia de trabajar la igualdad de género como tema transversal.

Una vez que esta actividad se había realizado, se procedió a utilizar en el aula el video del discurso pronunciado por Emma Watson el 20 de septiembre de 2014 en la ciudad de Nueva York para presentar la campaña HeforShe ${ }^{2}$ ante la asamblea de las Naciones Unidas. Esta campaña pretende empoderar a las mujeres y avanzar en la igualdad de género a nivel mundial. De esta forma se introducen recursos multimodales en el proceso de enseñanza-aprendizaje. Esta actriz y modelo británica señalaba en dicho video la necesidad de que exista igualdad económica, política y social entre ambos sexos, de modo que se pueda avanzar en derechos y libertades.

La profesora preguntó al estudiantado si conocían esta campaña para la igualdad de género promovida por ONU Mujeres una vez que el estudiantado había visto el video, de modo que se llevaba a cabo un ejercicio de escucha. Seguidamente, la profesora enfatizó que el fin de esta campaña era tomar conciencia de que es necesario avanzar en la igualdad de género para que tanto mujeres como hombres puedan asumir compromisos que contribuyan a acabar con las desigualdades de género.

Seguidamente, a la mitad de los grupos se les pide que busquen textos multimodales en la prensa relacionados con la violencia de género y la otra mitad ha de centrarse en encontrar textos de la publicidad en los que las mujeres sean empleadas como reclamo publicitario. Cada miembro del grupo ha de aportar al menos un texto. Todos los textos se han de poner en común

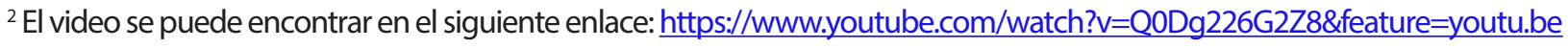


doi: http://dx.doi.org/10.15359/ree.23-2.15

URL: http://www.una.ac.cr/educare

CORREO: educare@una.cr

y se han de analizar teniendo en cuenta los principios de la gramática visual presentados en clase. Se ha de determinar si se observan algunos rasgos sistemáticos en la representación de las mujeres y establecer una unión entre estos y posibles estereotipos de género. Una vez que se lleva a cabo el análisis en los pequeños grupos, cada grupo ha de escribir un párrafo que resuma las características principales de los textos analizados.

Antes de compartir con toda la clase el análisis y las conclusiones a las que cada grupo llega, se nombra un portavoz en cada pequeño grupo y la profesora pide que se junten los distintos grupos que han trabajado sobre violencia de género por un lado y por otro lado se juntan los que han trabajado sobre la representación de las mujeres en la publicidad. Se selecciona un nuevo estudiante que tome nota de las ideas fundamentales que los grupos tienen en común y cuáles son diferentes con el fin de observar si son más las similitudes o las diferencias en el análisis. Cada portavoz presenta las principales ideas discutidas en su grupo. Cuando todos los grupos han participado, quien ha tomado nota esquematiza las principales ideas presentadas. A continuación, se establece un debate sobre la representación de las mujeres en los textos sobre violencia de género (estrategias de victimización, pasividad, etc.) y en los textos sobre publicidad (cosificación de los cuerpos de las mujeres, la mujer como reclamo publicitario, etc.).

Al final del cuatrimestre, cada estudiante tenía que buscar un texto multimodal sobre cualquier aspecto relacionado con el género. Cada alumna o alumno, de manera individual, tenía que hacer un breve esquema con los principales aspectos de la gramática visual que observaba en el texto. Finalmente, tenía que escribir una carta formal bien al periódico, bien a la agencia de publicidad o a cualquier otro lugar para pedir que cambiara el modo en que las mujeres son representadas en los medios. Dicha carta tenía que basarse en el texto que se había seleccionado y en todas las ideas discutidas en clase relacionadas con este tema.

La profesora enfatizó la necesidad de emplear un registro formal en el texto a la vez que se tenían en cuenta los recursos cohesivos explicados al principio del cuatrimestre. La profesora recogió todas las cartas y las devolvió corregidas en la clase siguiente. En las correcciones se prestó atención tanto a aspectos formales relacionados con la estructura del texto, el empleo de un vocabulario y unas estructuras gramaticales adecuadas para un registro formal. También se observó el uso de distintos recursos cohesivos y su relación con la relación clara de las ideas.

Como se puede ver, las distintas actividades relacionadas con cuestiones de género presentadas en esta sección se trabajan distintas destrezas: el estudiantado tiene que leer los textos que selecciona, tiene que presentar sus ideas delante de sus pares, tiene que escuchar los distintos puntos de visto de las demás personas y escuchar el video, establece interacciones dentro del grupo y entre los distintos grupos y, finalmente, tiene que escribir un texto formal.

\section{Discusión}

Las actividades presentadas en el apartado anterior permiten al estudiantado integrar las distintas destrezas que se han de trabajar al aprender una lengua extranjera (escucha,

María Martínez-Lirola

Los artículos de la Revista Electrónica Educare del Centro de Investigación y Docencia en Educación de la Universidad Nacional, Costa Rica, se comparten bajo términos de la Licencia Creative Commons: Reconocimiento, № Comercial, Sin Obra Derivada 3.0 Costa Rica. Las autorizaciones adicionales a las aquí delimitadas se pueden obtener en el correo: educare@una.cr 
interacción, escritura, habla y lectura). Además, se potencia la creatividad, pues el estudiantado tenía que tomar decisiones sobre los textos que seleccionaba para escribir el ensayo académico o sobre la carta formal que tenía que escribir.

Las dos temáticas seleccionadas se relacionan con algunos de los problemas que tiene la sociedad y con las propuestas de los objetivos del desarrollo sostenible. Sin duda, algunos de estos problemas tienen que ver con la discriminación por raza y con la violencia de género. Diseñar actividades que se refieran a estos retos sociales contribuye a que el estudiantado tome conciencia y asuma un papel activo para que, dentro de sus posibilidades, pueda ayudar a concienciar, transformar o acabar con dichos problemas, a la vez que se forma como ciudadanía global. El hecho de que el estudiantado tenga que analizar textos multimodales relacionados con la etnia y el género facilita la comprensión y la capacidad crítica sobre temas globales, y favorece el desarrollo de su conciencia social (Martínez-Lirola, 2013).

La primera actividad contribuye a que se rechace el racismo y otras formas de discriminación al promover la integración y la diversidad como características fundamentales de la sociedad del siglo XXI. Por tanto, se favorece la apertura a otras culturas, y el respeto y el valor de las diferencias culturales con el fin de desterrar la idea de que hay culturas superiores a otras. La segunda actividad contribuye a potenciar la igualdad de oportunidades entre mujeres y hombres, y a visibilizar las diferencias que contribuyen a la discriminación de las mujeres, prestando especial atención a la violencia de género.

El tipo de actividades descritas en este artículo se usa en la clase de inglés como lengua extranjera con el fin de introducir temas globales como la etnia o el género y también con la finalidad de trabajar con textos auténticos. Seleccionar textos multimodales auténticos contribuye a trabajar temas sociales en el aula de modo que se pueda establecer una conexión entre lo que ocurre en las clases y en la sociedad. Por tanto, se potencia que la universidad del siglo XXI se centre no solo en la enseñanza de contenidos, sino también en competencias como la conciencia social, el respecto a la diversidad, la resolución de conflictos o el liderazgo.

El hecho de enseñar un curso de lengua inglesa a estudiantes con un nivel avanzado ofrece la posibilidad de elegir varios temas globales para trabajar con el vocabulario de varios campos semánticos, en distintas construcciones gramaticales y en la discusión de temas. Cuando el profesorado selecciona temas de contenido social tiene como objetivo mejorar la capacidad crítica del estudiantado. Esto es posible porque el análisis de los textos seleccionados contribuye a que sus estudiantes reflexionen sobre las causas y las consecuencias que hay detrás de los distintos temas que se presentan en los textos, como por ejemplo, los intereses económicos, el poder, la discriminación, la injusticia, etc. (Martínez-Lirola, 2013).

Las actividades descritas potencian la adquisición de las competencias sociales enumeradas en la sección primera de este artículo, entre las que destacan: la comunicación, pues en todo momento del proceso de enseñanza-aprendizaje el estudiantado tiene que hablar 
doi: http://dx.doi.org/10.15359/ree.23-2.15

URL: http://www.una.ac.cr/educare

CORREO: educare@una.cr

con sus pares o escribir; la cooperación, al tener que colaborar en los grupos para lograr realizar las actividades como el ensayo o el glosario cooperativo. También se trabaja la empatía, al tener que reflexionar sobre realidades sociales difíciles relacionadas con el racismo o el género que afectan a muchas personas.

De hecho, en esta propuesta didáctica el aprendizaje es efectivo y significativo porque no se centra solo en los contenidos que se han de enseñar siguiendo el currículo, sino que también se potencia la adquisición de competencias diversas que sean útiles tanto para el mercado laboral como para la vida personal. Además, se promueve la conciencia social debido a que las distintas actividades propuestas hacen que el estudiantado tome conciencia de realidades sociales distintas de la propia, pueda comprender la situación de distintas personas y sentir que son ciudadanía activa en una sociedad globalizada, donde las diferencias individuales y culturales han de ser tenidas en cuenta.

La combinación de temas globales y una propuesta didáctica basada en competencias como la presentada en este artículo ofrece la posibilidad de conceder importancia a la diversidad y de presentar distintas perspectivas en la clase de idiomas (Bajaj, 2008). Son diversos los efectos positivos de trabajar con temas globales en el aula debido a que el estudiantado participa de manera activa, se implica en todo el proceso de enseñanza-aprendizaje y tiene motivación para llevar a cabo las actividades.

Introducir el análisis de textos con contenido social en el aula contribuye a que sus estudiantes tomen conciencia de la necesidad de llevar a cabo transformaciones sociales en el mundo y de asumir un papel activo y crítico como ciudadanía del siglo XXI (Barahona et al., 2013). La selección de textos relacionados con el racismo y el género también es útil para trabajar con el estudiantado sobre los prejuicios que muchas personas tienen contra las personas de otras etnias, sobre la discriminación por género y para discutir los beneficios de la diversidad cultural.

Las diferentes actividades presentadas en las secciones anteriores pretenden ayudar al estudiantado a pensar sobre distintos modos de discriminar a los seres humanos (por raza y por género). En consecuencia, se destaca la importancia de acabar con la discriminación en todas sus formas, con el fin de tratar a todas las personas con respeto e igualdad con independencia de la raza, el sexo o la clase social que se tenga.

Organizar la asignatura Lengua Inglesa $V$ en torno a profundizar en temas sociales como el racismo y el género contribuye a introducir temas relacionados con los derechos humanos y la justicia en una clase de lengua extranjera. De esta forma, el currículo hace explícita la importancia de los temas sociales y su relación con los valores y las competencias sociales. De hecho, enseñar contenidos lleva consigo enseñar competencias y conceder importancia a la naturaleza social de la educación. En este sentido, se invita a estudiantes a observar sus sentimientos al trabajar con los textos seleccionados. En consecuencia, se presta atención no 
solo al contenido lingüístico y visual de los textos, sino también al potencial emocional que tienen y que el estudiantado debe observar en el análisis.

Además, elegir temas sociales de actualidad y diseñar actividades que combinen el desarrollo de las destrezas orales y escritas concede al estudiantado la oportunidad de participar activamente durante el proceso de enseñanza-aprendizaje, a la vez que desarrollan la conciencia de ser ciudadanía activa en el siglo XXI. El hecho de que el estudiantado tenga que discutir con sus compañeras y compañeros y expresar sus opiniones sobre las distintas realidades que se describen en los textos lleva consigo poner en práctica distintas competencias sociales, como el respecto a la diversidad de opiniones o la escucha activa.

Con el fin de obtener información cuantitativa que apoyara la información cualitativa que se ha presentado en los párrafos anteriores, al final del cuatrimestre, se preparó una encuesta para conocer la opinión del estudiantado sobre algunos aspectos pedagógicos del proceso de enseñanza-aprendizaje y para saber si las actividades propuestas contribuyen a su formación como ciudadanía global. La encuesta fue anónima y la contestó todo el estudiantado que había asistido a clase y había participado en las actividades descritas.

La primera pregunta trababa de saber si el estudiantado consideraba que en la universidad se deben enseñar solo contenidos o también se ha de trabajar en la adquisición de competencias. Un 7\% respondió que solo contenidos frente a un 93\% que contestó que también se habían de adquirir competencias además de contenidos.

Seguidamente, se pretendía saber si el estudiantado consideraba que trabajar con textos reales en el aula le permitía acercarse y conocer distintas realidades sociales. Un 91 \% contestó que sí frente a un $9 \%$ que ofreció una respuesta negativa. La pregunta tercera planteaba si al estudiantado le parecía importante introducir distintas problemáticas sociales en el proceso de enseñanza aprendizaje. La gran mayoría, el 93 \% responde que sí frente al 7 \% que responde lo contrario. Como se puede ver, en esta respuesta encontramos el mismo porcentaje en las respuestas que en la pregunta primera, hecho que nos lleva a plantearnos la posibilidad de que fuera el mismo estudiantado el respondiera sí y no en ambas preguntas.

En esta pregunta también se pretendía saber en las razones que tenía el estudiantado para optar por una u otra respuesta. Por un lado, cuando la respuesta fue afirmativa, las principales razones que dieron para justificar su respuesta fueron las siguientes: porque así profundizo en lo que está pasando en la sociedad; para establecer una unión entre lo que se enseña en el aula y lo que ocurre en la vida, o para poder reflexionar sobre lo que está ocurriendo y poder contribuir a ofrecer alguna solución. Por otro lado, cuando la respuesta fue negativa, las razones presentadas fueron: porque considero que lo importante en esta asignatura es aprender inglés o porque lo más importante es profundizar en aspectos gramaticales. 
doi: http://dx.doi.org/10.15359/ree.23-2.15

URL: http://www.una.ac.cr/educare

CORREO: educare@una.cr

Con la pregunta 4 simplemente se pretendía saber si el estudiantado conocía los objetivos del desarrollo sostenible antes de que la profesora los mencionara en esta asignatura. El $48 \%$ respondió que sí frente a un $52 \%$ que respondió que no. A continuación, se preguntó si el aprendizaje cooperativo empleado en el proceso de enseñanza-aprendizaje había ayudado al estudiantado a aprender mejor. Un 86 \% respondió que sí y justificó su respuesta por medio de los argumentos que siguen: porque siempre es más interesante y motivador trabajar con otras personas; porque se potencia la interacción, porque se fomenta conocer otros puntos de vista y otras maneras de hacer las cosas y porque todas las personas contribuyen a la realización de la tarea y, por lo tanto, se sienten responsables y motivadas para colaborar. El 14,\% que ofreció una respuesta negativa argumentó que su respuesta se basaba en que tardaba más haciendo las tareas con otras personas y en que a veces surgen conflictos en el grupo que dificultan llevar a cabo las tareas.

Con la pregunta 6 se preguntaba al estudiantado si las actividades realizadas habían contribuido a que adquiriera competencias sociales. El 100,\% del estudiantado ofreció una respuesta positiva y la justificaron de la siguiente manera: tomando más conciencia de determinados problemas sociales como la violencia de género o el racismo; comprendiendo realidades sociales distintas de la propia; favoreciendo actitudes de solidaridad y de apertura ante las realidades sociales de distintas personas; apostando por la creación de un mundo más justo y equitativo en el que todas las personas vivan con dignidad; reflexionando sobre el modo en que la educación superior nos puede acercar a distintas realidades sociales a través de los materiales que se empleen y avanzando como ciudadanía crítica, comprometida y responsable de la construcción de un mundo mejor y a la transformación social.

Finalmente, con la pregunta 7 se pretendía saber si el alumnado consideraba que la temática social de las actividades realizadas había contribuido a su formación como ciudadanía global. La inmensa mayoría, en concreto un $94 \%$, eligió una respuesta afirmativa. La mayor parte de las respuestas que el alumnado ofrece para justificar su elección señalan que, gracias a las actividades realizadas, son conscientes de realidades sociales distintas de la suya y de las transformaciones sociales que es necesario llevar a cabo para que la sociedad avance en justicia, igualdad y respeto a la diversidad, entre otros. Además, destacan que han reflexionado sobre distintos aspectos culturales y han tomado conciencia de la importancia que tiene la cultura en la evolución de cada ser humano. En concreto, el $62 \%$ del alumnado que responde positivamente afirma que gracias a las actividades realizadas ha sido capaz de empatizar con las situaciones injustas que viven otras personas, hecho que les ha permitido superar su visión etnocéntrica del mundo.

\section{Conclusiones}

La propuesta de enseñanza presentada en este artículo destaca la importancia de emplear textos de contenido social en la enseñanza de modo que se pueda favorecer la adquisición de competencias sociales, al potenciar la relación entre la temática de los textos y la realidad social. 
Tanto los materiales como los temas para la discusión se seleccionaron teniendo en cuenta su contenido social, con el fin de establecer relaciones entre la enseñanza y la sociedad. De esta forma, se concede importancia a que el estudiantado mejore su conciencia social y su capacidad crítica de modo que avance como ciudadanía activa en el siglo XXI.

La cooperación ocupa un lugar central en el proceso de enseñanza-aprendizaje, pues el estudiantado trabaja con sus compañeras y compañeros, con el fin de realizar las actividades y lograr un objetivo común. Además, el estudiantado adquiere competencias diversas, incluidas las sociales, a la vez que aprende lengua inglesa. Basar el proceso de enseñanza-aprendizaje en el aprendizaje cooperativo y en el empleo de textos reales contribuye a fomentar la interacción y el diálogo.

Conceder importancia a una propuesta de enseñanza basada en la adquisición de competencias además de en contenidos lleva consigo que lo que se enseña y se aprende en las aulas universitarias debe tener una relación directa con las demandas de la sociedad actual y, específicamente, con las demandas del mercado laboral. Las actividades y la metodología propuestas muestran que la asignatura se centra en el estudiantado en lugar del profesorado, es decir, el estudiantado es el protagonista del proceso de enseñanza-aprendizaje. En consecuencia, se potencia una relación igualitaria entre el profesorado y el estudiantado, pues ambos comparten la responsabilidad.

El hecho de que la asignatura esté basada en contenidos sociales facilita el desarrollo de la conciencia social y la responsabilidad del estudiantado, porque toma conciencia de distintas realidades sociales y observa ejemplos de desigualdad social y de injusticia en algunos casos. En consecuencia, los grupos de estudiantes avanzan en su proceso de ser ciudadanía global al abrirse a realidades sociales distintas de la propia.

Este artículo muestra que planificar una asignatura sobre la base de contenidos sociales y seleccionar textos que los refuercen no solo contribuye a la adquisición de competencias, sino también a aumentar la formación social y global del alumnado. En este sentido, es fundamental diseñar actividades en la clase de inglés como lengua extranjera en las que se conceda importancia tanto a los contenidos como a las competencias sociales, tales como la empatía o el respeto a la diversidad

\section{Referencias}

Baesler, E. J. y Lauricella, S. (2014). Teach peace: Assessing instruction of the nonviolent communication and peace course. Journal of Peace Education, 11(1), 46-63. doi: https:// doi.org/10.1080/17400201.2013.777899

Bajaj, M. (2008). Introduction. En M. Bajaj (Ed.), Encyclopedia of peace education (pp. 1-11). Charlotte, North Carolina: Information Age Publishing. 
doi: http://dx.doi.org/10.15359/ree.23-2.15

URL: http://www.una.ac.cr/educare

CORREO: educare@una.cr

Barahona, R., Gratacós, J. y Quintana, G. (2013). Centros educativos transformadores. Ciudadanía global y transformación social. Barcelona: Oxfam Intermón.

Bisquerra, R. y Pérez, N. (2007). Las competencias emocionales. Educación XXI, 10, 61-82. doi: https://doi.org/10.5944/educxx1.1.10.297

Boni, A. y Calabuig, C. (2017). Education for global citizenship at Universities. Journal of Studies in International Education, 21(1), 22-38. doi: https://doi.org/10.1177/1028315315602926

Boni, A., Lopez-Fogues, A. y Walker, M. (2016). Higher education and the post-2015 agenda: A contribution from the human development approach. Journal of Global Ethics, 12(1), 1728. doi: https://doi.org/10.1080/17449626.2016.1148757

Cantón Mayo, I., Valle Flórez, R. E., Arias Gago, A. R., Baelo Álvarez, R. y Cañón Rodríguez, R. (Coords.). (2010). Calidad, comunicación e interculturalidad. Barcelona: Davinci Continental.

Consejo de Europa. (2001). Marco Común Europeo de Referencia de las Lenguas. Cambridge: Cambridge University Press.

De Sylva Joyce, H. y Feez, S. (2012). Text-based language and literacy education: Programming and methodology. Sidney: Phoenix Education.

Francisco, A. (2010). Construyendo ciudadanía participativa en la sociedad de la información. Pixel Bit. Revista de Medios y Educación, 37, 135-146. Recuperado de https://recyt.fecyt.es/ index.php/pixel/article/view/61405/37418

Johnson, D.W. y Johnson, F.P. (2009). Joining together: Group theory and group skills (10a ed.). Boston: Allyn and Bacon.

Johnson, D.W., Johnson, R. T. y Holubec, E. (1998). Cooperation in the classroom. Boston: Allyn and Bacon.

Johnson, D. W. y Johnson, R. T. (2009). Energizing learning: The instructional power of conflict. Educational Researcher, 38(1), 37-51. doi: https://doi.org/10.3102/0013189X08330540

Kress, G. y van Leeuwen, T. (2006). Reading images: The grammar of visual design (2ª ed.). Londres: Routledge.

Kruger, F. (2012). The role of TESOL in educating for peace. Journal of Peace Education, 9(1), 17-30. doi: https://doi.org/10.1080/17400201.2011.623769

Martin, J. R. y Rose, D. (2012). Learning to write/learning to learn: Genre, knowledge and pedagogy in the Sydney School. Londres: Equinox. 
Martínez-Lirola, M. (2013). La relación entre el aprendizaje cooperativo y la adquisición de competencias interpersonales en una clase de lengua inglesa. Encuentro. Revista de investigación e innovación en la clase de idiomas, 22, 73-83. Recuperado de https:// ebuah.uah.es/dspace/bitstream/handle/10017/19946/relaci\%c3\%b3n Martinez Encuentro 2013 N22.pdf?sequence=1\&isAllowed $=y$

Martínez-Lirola, M. (2014). Propuesta de enseñanza multimodal en una asignatura de lengua inglesa. Encuentro. Revista de investigación e innovación en la clase de idiomas, 23, 89-101. Recuperado de https://ebuah.uah.es/dspace/handle/10017/21595

Moss, G., Barletta, N., Chamorro, D., y Mizuno, J. (2015). Educating citizens in the foreign language classroom: Missed opportunities in a Colombian EFL textbook. En X. L. Curdt-Christiansen y C. Weninger (Eds.), Language, Ideology and Education. The Politics of Textbooks in Language Education (pp. 69-89). Londres: Routledge.

Porto, M. (2015). Developing intercultural citizenship education in the language classroom and beyond. Argentinian Journal of Applied Linguistics, 3(2), 9-29. Recuperado de http://www. faapi.org.ar/ajal/issues/302/PortoAJALVol3(2).pdf

Priegue, D. y Leiva, J. J. (2012). Las competencias interculturales en la sociedad del conocimiento: Reflexiones y análisis pedagógico. EDUTEC. Revista Electrónica de Tecnología Educativa, 40, 1-12. doi: https://doi.org/10.21556/edutec.2012.40.370

Prieto, L. (2007). El aprendizaje cooperativo. Madrid: PPC.

Rosales, C. (2015). Los temas transversales en el aula. Convivencia, salud e igualdad. Santiago de Compostela: Andavira.

Saura, D. y Del Valle Antolín, J. (2012). Implantación del modelo de aprendizaje cooperativo en secundaria obligatoria. Colegio La Salle de Palencia. En A. Hernández y S. Olmos (Eds.), Metodologías de aprendizaje cooperativo a través de las tecnologías (pp. 85-94). Salamanca: Aquilafuente.

Tallón, R. y Sikora, M. (2011). Conciencia en acción. Eneagrama, inteligencia emocional y cambio (Trad. N. Steinbrun). Madrid: Alquimia.

Vinagre, M. (2014). El desarrollo de la competencia intercultural en los intercambios telecolaborativos. RED, Revista de Educación a Distancia, 41, 1-22. Recuperado de http:// www.um.es/ead/red/41/vinagre.pdf

Wang, N., Young, T. Wilhite, S. C. y Marczyk, G. (2011). Assessing students' emotional competence in Higher Education: Development and validation of the widener emotional learning scale. Journal of Psychoeducational Assessment, 29(1), 47-62. doi: https://doi. org/10.1177/0734282909359394 
doi: http://dx.doi.org/10.15359/ree.23-2.15

URL: http://www.una.ac.cr/educare

CORREO: educare@una.cr

Apéndice A: Encuesta sobre el proceso de enseñanza-aprendizaje

1. ¿Consideras que en la universidad se han de enseñar solo contenidos o también se ha de trabajar en la adquisición de competencias?

Solo contenidos

También competencias además de contenidos

2. ¿Piensas que trabajar con textos reales contribuye a que te acerques a distintas realidades sociales?<smiles>[Si]CC=[Si]</smiles>

3. ¿Te parece importante introducir distintas problemáticas sociales en el proceso de enseñanza aprendizaje? Sí No

Si la respuesta es afirmativa, ¿por qué?

Si la respuesta en negativa, ¿por qué?

4. ¿Conocías los objetivos del desarrollo sostenible antes de que la profesora los mencionara en esta asignatura?

Sí

No

5. ¿Te ha ayudado el aprendizaje cooperativo empleado en el proceso de enseñanza-aprendizaje a aprender mejor?

Sí

No

Si la respuesta es afirmativa, ¿por qué?

Si la respuesta en negativa, ¿por qué?

6. En tu opinión, ¿han contribuido las actividades realizadas a la adquisición de competencias sociales?

Si

No

Si la respuesta es afirmativa, ¿de qué forma?

Si la respuesta en negativa, ¿por qué?

7. ¿Consideras que la temática social de las actividades que has realizado ha contribuido a tu formación como ciudadanía global?

Sí _ No

¿Por qué? 